\title{
Fishing for a better language
}

Stuart Schwartz, PhD

For most school children, sometime either in elementary school or middle school, they are informed that they need to begin to learn a second language, one different from their native tongue. Although some will always continue to remember and use the second language, many forget most of what they are taught the moment they leave high school. Studies have shown that students are most adept at learning languages when they are younger and as you become older it becomes more difficult to master the new language. Unfortunately for cytogeneticists, we don't begin to learn our new language, the language of cytogenetic nomenclature until our 20's and 30's. The language came about in 1960 at the Denver Conference, as it became obvious that there was a need to be able to describe cytogenetic results, especially abnormal findings. ${ }^{1}$ It was essential to have the ability to transmit results from lab to lab and from lab to physician. This nomenclature became modified over the next 10 years, and after the initiation of banding, refined at the Paris Conference in $1971 .^{2}$ Most of us have heard multiple stories of the initiation and refinement of this language and terminology, such as the origin of the short (p) arm and long (q) arm. It is essential, however, to realize what a daunting task it was to start when there was no information and to build a language from scratch. It is probably the most remarkable achievement in language since Tolkien developed multiple languages for Lord of the Rings. We owe an immeasurable debt to all those scientists involved in the initiation and development of the nomenclature. This nomenclature has undergone continual changes over the past 30 years, with the most recent in 1995, through the International Standing Committee for Cytogenetic Nomenclature, when the ISCN 1995 publication first included FISH (fluorescence in situ hybridization) nomenclature. ${ }^{3}$

As detailed by Mascarello et al. ${ }^{4}$ in this issue of Genetics in Medicine, the FISH nomenclature introduced in 1995 has not stood up as well, as has the original conventional cytogenetic nomenclature. Through the use of 7 different CAP (College of Pathologists) surveys involving FISH, they analyzed 15 challenges to determine how effectively and consistently the FISH nomenclature was used. From these challenges, the CAP resource committee, who knew that the current nomenclature works exceedingly well with conventional cytogenetics to describe abnormalities, wanted to test if the nomenclature also worked with the utilization of FISH. Surprisingly, they showed that there was incorrect FISH syntax in $11 \%$ to $49 \%$ of the responses from the participating laboratories. More specifically, they showed that overall, the laboratories did better with

Case Western Reserve University, and University Hospitals of Cleveland, Department of Genetics, Center for Human Genetics, 10524 Euclid Ave, Cleveland OH 44106-9959. microdeletions than with either aneuploidy interphase descriptions or interphase cancer descriptions. They indicated that in only 5 of 14 challenges that they tried to score was the $80 \%$ consensus criteria met to allow scoring a challenge by the CAP resource committee. This number is much lower than that for the conventional cytogenetic challenges. It is somewhat surprising that there was difficulty by laboratories with both the SNRPN probes and X and Y probes in transplants, as delineated by these authors. However, it is not at all surprising that the laboratories had difficulties with probes studying BCR/Abl and Tel/AML1 fusions. For the detection of the BCR/ Abl fusion, there are several different probe sets used to detect the fusion of these two genes. Each of these probe sets will give different interphase FISH patterns, making it more difficult to develop a consistent way of describing the fusion. Additionally, when using the BCR/Abl and Tel/AML1 probe sets to detect fusions, even when the fusion is present, there is sometimes a concomitant deletion of either part of the gene or possibly the entire gene, thus making the description by FISH analysis even more difficult. These are only a few examples, but they certainly illustrate some of the problems regarding the FISH nomenclature as it currently exists. In addition, with the sequencing of the majority of the human genome, many more unique sequence probes can and will be used to describe cytogenetic structure, also complicating nomenclature as multiple probes might be used for its ultimate description.

In a review of their study, Mascarello et al. ${ }^{4}$ noted that the current FISH nomenclature was not suitable for Proficiency Test purposes, impractical for database management, but most importantly impractical for clinical test reporting. The CAP resource committee has done a superb first step in identifying and quantifying a problem in the utilization of the current nomenclature when FISH is used. It is a challenge for the ISCN Committee to improve upon the current nomenclature that is consistent and able to describe all types of abnormalities. The CAP resources stated, "it is the value of such a system for communicating observations between Geneticists and for cataloging cytogenetic data in a manner that readily fosters correlation with clinical observation." ${ }^{\prime 4}$ In order to better achieve this goal and improve the limitations of the nomenclature, they have suggested to expand nomenclature guidelines by setting standards for the following: (1) probe designation; (2) band level for probes target; (3) when low-frequency cell types should be included; (4) order of normal cell lines in bone marrow transplant patients; (5) inclusion of control probes; and (6) listing of $\mathrm{Y}$ chromosome probes in XX nuclei. Additionally, they indicate that they believe that not all of the information currently in the karyotypes should in fact be listed in the karyotypes. For example, they believe that the probe names, rather than being 
part of the karyotype should be listed in the reports, just as the banding is (e.g., G-banding). All of their criticisms and suggestions in the manuscript are valid and thought provoking. However, as we are all familiar with, every change that is made, in any system, does not always have its intended consequence and may not always improve the situation. For example, although removing the probe name from the karyotype will make the nomenclature less cumbersome, if a lab sends a sample to another lab for analysis, they may only report the karyotype and not the additional information. It is therefore incumbent on the ISCN Committee to evaluate these suggestions as well as other obtained from workers in the field to determine how best the nomenclature can be altered. As detailed above, this will not be an easy task, as many probes can have a number of findings and many uses, many not originally intended. Additionally, not only will this committee need to review all of the FISH nomenclature currently used, but they should become proactive and review new probes as they become released by companies or investigators and develop appropriate nomenclature as the probes are put into use. This will not be an easy task due to the number of probes currently available, their varied uses, and the frequency with which more probes are released. However, a systematic approach to all the probes is needed to develop more consistency in the nomenclature.

The CAP proficiency tests are an excellent vehicle to continue to test the effectiveness of the nomenclature that we are using. It is obviously premature to test the use of this nomen- clature in a graded system, but as refinements are made, the effectiveness of these changes certainly can be continued to be tested as ungraded challenges in these tests to monitor how well everyone is doing with the nomenclature. Over the years, the scientists in our field have done excellent work with keeping abreast of changes and modifying both methodology and description of the abnormal findings. There is no doubt the ISCN Committee will be able to make the appropriate modification needed. Lets hope that within the next couple of years, we will no longer be fishing for a language, but will rather have hooked improvements that can be cast out to the scientific Genetic community.

Stuart Schwartz, PhD

From the Case Western Reserve University, and University Hospitals of Cleveland, Department of Genetics, Center for Human Genetics, Cleveland, Ohio

\section{Reference}

1. Denver Conference. A proposed standard system of nomenclature of human mitotic chromosomes. Lancet 1960;I:1063-1065.

2. Paris Conference (1971). Standardization in human cytogenetics. Birth defects: Original article series, vol 8, no 7. New York: The National Foundation; 1972.

3. International Standing Committee of Human Cytogenetic Nomenclature; Mitelman F, editor. An International System for Human Cytogenetic Nomenclature. Basal, Switzerland: S. Karger AG; 1995.

4. Mascarello JT, Cooley LD, Davison K, Dewald GW, Brothman AR, Herrman M et al. Problems with ISCN FISH nomenclature make it not practical for use in clinical test reports or cytogenetic databases. Genet Med 2003;5:370-377. 